Network Working Group

Request for Comments: 1794

Category: Informational
T. Brisco

Rutgers University

April 1995

\title{
DNS Support for Load Balancing
}

Status of this Memo

This memo provides information for the Internet community. This memo does not specify an Internet standard of any kind. Distribution of this memo is unlimited.

1. Introduction

This RFC is meant to first chronicle a foray into the IETF DNS Working Group, discuss other possible alternatives to provide/simulate load balancing support for DNS, and to provide an ultimate, flexible solution for providing DNS support for balancing loads of many types.

2. History

The history of this probably dates back well before my own time - so undoubtedly some holes are here. Hopefully they can be filled in by other authors.

Initially; "load balancing" was intended to permit the Domain Name System (DNS) [1] agents to support the concept of "clusters" (derived from the VMS usage) of machines - where all machines were functionally similar or the same, and it didn't particularly matter which machine was picked - as long as the load of the processing was reasonably well distributed across a series of actual different hosts. Around 1986 a number of different schemes started surfacing as hacks to the Berkeley Internet Name Domain server (BIND) distribution. Probably the most widely distributed of these were the "Shuffle Address" (SA) modifications by Bryan Beecher, or possibly Marshall Rose's "Round Robin" code.

The SA records, however, did a round-robin ordering of the Address resource records, and didn't do much with regard to the particular loads on the target machines. Matt Madison (of TGV) implemented some changes that used VMS facilities to review the system loads, and return A RRs in the order of least-loaded to most loaded.

The problem was with SAs was that load was not actually a factor, and $\mathrm{TGV}^{\prime} \mathrm{S}$ relied on VMS specific facilities to order the records. The SA RRs required changes to the DNS specification (in file syntax and in 
record processing). These were both viewed as drawbacks and not as general solutions.

Most of the Internet waited in anticipation of an IETF approved method for simulating "clusters".

Through a few IETF DNS Working Group sessions (Chaired by Rob Austein of Epilogue), it was collectively agreed upon that a number of criteria must be met:

A) Backwards compatibility with the existing DNS RFC.

B) Information changes frequently.

C) Multiple addresses should be sent out.

D) Must interact with other RRs appropriately.

E) Must be able to represent many types of "loads"

F) Must be fast.

(A) would ensure that the installed base of BIND and other DNS implementations would continue to operate and interoperate properly.

(B) would permit very fast update times - to enable modeling of real-time data. Five minutes was thought as a normal interval, though changes as fast as every sixty seconds could be imagined.

(C) would cover the possibility of a host's address being advertised as optimal, yet the machine crashed during the period within the TTL of the RR. The second-most preferable address would be advertised second, the third-most preferable third, and so on. This would allow a reasonable stab at recovery during machine failures.

(D) would ensure correct handling of all ancillary information - such as MX, RP, and TXT information, as well as reverse lookup information. It needed to be ensured that such processes as mail handling continued to work in an unsurprising and predictable manner.

(E) would ensure the flexibility that everyone wished. A breadth of "loads" were wished to be represented by various members of the DNS Working Group. Some "loads" were fairly eclectic - such as the address ordering by the RTT to the host, some were pragmatic - such as balancing the CPU load evenly across a series of hosts. All represented valid concerns within their own context, and the idea of having separate $R R$ types for each was unthinkable (primarily; it would violate goal A). 
(F) needed to ensure a few things. Primarily that the time to calculate the information to order the addressing information did not exceed the TTL of the information distributed - i.e., that elements with a TTL of five minutes didn't take six minutes to calculate. Similarly; it seems a fairly clear goal in the DNS RFC that clients should not be kept waiting - that request processing should continue regardless of the state of any other processing occurring.

\section{Possible Alternatives}

During various discussions with the DNS Working Group and with the Load Balancing Committee, it was noted that no existing solution dealt with all wishes appropriately. One of the major successes of the DNS is its flexibility - and it was felt that this needed to be retained in all aspects. It was conceived that perhaps not only address information would need to be changed rapidly, but other records may also need to change rapidly (at least this could not be ruled out - who knows what technologies lurk in the future).

Of primary concern to many was the ability to interact with older implementations of DNS. The DNS is implemented widely now, and changes to critical portions of the protocol could cause havoc for years. It became rapidly apparent through conversations with Jon Postel and Dave Crocker (Area Director) that modifications to the protocol would be viewed dimly.

4. A Flexible Model

During many hours of discussions, it arose upon suggestion from Rob Austein that the changes could be implemented without changes to the protocol; if zone transfer behavior could be subtly changed, then the zone transfer process could accommodate the changing of various RR information. What was needed was a smarter program to do the zone transfers. Pursuant to this, changes were made to BIND that would permit the specification of the program to do the zone transfers for particular zones.

There is no specification that a secondary has to receive updates from its primary server in any specific manner - only that it needs to check periodically, and obtain new zone copies when changes have been made. Conceivably the zone transfer agent could obtain the information from any number of sources (e.g., a load average daemon, a round-robin sorter) and present the information back to the nameserver for distribution.

A number of questions arose from this concept, and all seem to have been dealt with accordingly. Primarily, the DNS protocol doesn't guarantee ordering. While the DNS protocol doesn't guarantee 
ordering, it is clear that the ordering is predictive - that information read in twice in the same order will be presented twice in the same order to clients. Clients, of course, may reorder this information, but that is deemed as a "local issue" as it is configurable by the remote systems administrators (e.g., sortlists, etc). The zone transfer agent would have to account for any "misordering" that may occur locally, but remote reordering (e.g., client side sortlists) of RRs is is impossible to predict. Since local mis-ordering is consistent, the zone transfer agents could easily account for this.

Secondarily, but perhaps more subtly, the problem arises that zone transfers aren't used by primary nameservers, only by secondary nameservers. To clarify this, the idea of "fast" or "volatile" subzones must be dealt with. In a volatile environment (where address or other $R R$ ordering changes rapidly), the refresh rate of a zone must be set very low, and the TTL of the RRs handed out must similarly be very low. There is no use in handing out information with TTLs of an hour, when the conditions for ordering the RRs changes minutely. There must be a relatively close relationship between the refresh rates and TTLs of the information. Of course, with very low refresh rates, zone transfers between the primary and secondary would have to occur frequently. Given that primary and secondary nameservers should be topologically and geographically far apart, moving that much data that frequently is seen as prohibitive. Also; the longer the propagation time between the primary and secondary, the larger the window in which circumstances can change thus invalidating the secondary's information. It is generally thought that passing volatile information on to a secondary is fairly useless - if secondaries want accurate information, then they should calculate it themselves and not obtain it via zone transfers. This avoids the problem with secondaries losing contact with the primaries (but access to the targets of the volatile domain are still reachable), but the secondary has information that is growing stale.

What is essentially necessary is a secondary (with no primary) which can calculate the necessary ordering of the RR data for itself (which also avoids the problem of different versions of domain servers predictively ordering $R R$ information in different predictive fashions). For a volatile zone, there is no primary DNS agent, but rather a series of autonomous secondary agents. Each autonomous secondary agent is, of course, capable of calculating the ordering or content of the volatile RRs itself. 
5. Implementation

With some help from Masataka Ohta (Tokyo Institute of Technology), I implemented modifications to BIND to permit the specification of the zone transfer program (zone transfer agent) for particular domains:

transfer <domain-name> <program-name>

Currently I define a separate subdomain that has a few hosts defined in it - all volatile information. The zone has a refresh rate of 300, and a minimum TTL of 300 indicated. The configuration file is indicated as "volatile.hosts". Every 300 seconds a program "doAxfer" is run to do the zone transfer. The program "doAxfer" reads the file "volatile.hosts.template" and the file "volatile.hosts.list". The addresses specified in volatile.hosts.list are rotated a random number of times, and then substituted (in order) into volatile.hosts.template to generate the file volatile.hosts. The program "doAxfer" then exits with a value of 1 - to indicate to the nameserver that the zone transfer was successful, and that the file should be read in, and the information distributed. This results in a host having multiple addresses, and the addresses are randomized every five minutes (300 seconds).

Two bugs continue to plague us in this endeavor. BIND currently considers any TTL under 300 seconds as "irrational", and substitutes in the value of 300 instead. This greatly hampers the functionality of volatile zones. In the fastest of all cases - a 0 TTL information would be used once, and then thrown away. Presumably the new RR information could be calculated every 5 seconds, and the RRs handed out with a TTL of 0 . It must be considered that one limitation of the speed of a zone is going to be the ability of a machine to calculate new information fast enough.

The other bug that also effects this is that, as with TTLs, BIND considers any zone refresh rate under 15 minutes to be similarly irrational. Obviously zone refresh rates of 15 minutes is unacceptable for this sort of applications.

For a work-around, the current code sets these same hard-coded values to 60 seconds. Sixty seconds is still large enough to avoid any residual bugs associated with small timer values, but is also short enough to allow fast subzones to be of use.

This version of BIND is currently in release within Rutgers University, operating in both "fast" and normal zones. 


\section{Performance}

While the performance of fast zones isn't exactly stellar, it is not much more than the normal CPU loads induced by BIND. Testing was performed on a sun Sparc-2 being used as a normal workstation, but no resolvers were using the name server - essentially the nameserver was idle. For a configuration with no fast subzones, BIND accrued 11 CPU seconds in 24 hours. For a configuration with one fast zone, six address records, and being refreshed every 300 seconds (5 minutes), BIND accrued 1 minute 4 seconds CPU time. For the same previous configuration, but being refreshed every sixty seconds, BIND accrued 5 minutes and 38 seconds of CPU time.

As is no great surprise, the CPU load on the serving machine was linear to the frequency of the refresh time. The sixty second refresh configuration used approximately five times as much CPU time as did the 300 second refresh configuration. One can easily extrapolate that the overall CPU utilization would be linear to the number of zones and the frequency of the refresh period. All of this is based on a shell script that always indicated that a zone update was necessary, a more intelligent program should realize when the reordering of the RRs was unnecessary and avoid such periodic zone reloads.

7. Acknowledgments

Most of the ideas in this document are the results of conversations and proposals from many, many people - including, but not limited to, Robert Austein, Stuart Vance, Masataka Ohta, Marshall Rose, and the members of the IETF DNS Working Group.

8. References

[1] Mockapetris, P., "Domain Names - Implementation and Specification", STD 13, RFC 1035, USC/Information Sciences Institute, November 1987 . 
9. Security Considerations

Security issues are not discussed in this memo.

10. Author's Address

Thomas P. Brisco

Associate Director for Network Operations

Rutgers University

Computing Services, Telecommunications Division

Hill Center for the Mathematical Sciences

Busch Campus

Piscataway, New Jersey 08855-0879

USA

Phone: +1-908-445-2351

EMail: brisco@rutgers.edu 